\title{
Highlights from LHC experiments and future perspectives ${ }^{1}$
}

\author{
P. Campana \\ INFN - Laboratori Nazionali di Frascati, 00044 Frascati, Italy
}

\begin{abstract}
The experiments at LHC are collecting a large amount of data in a kinematic of the $\left(x, Q^{2}\right)$ variables never accessed before. Boosted by LHC analyses, Quantum Chromodynamics (QCD) is experiencing an impressive progress in the last few years, and even brighter perspectives can be foreseen for the future data taking. A subset of the most recent results from the LHC experiments in the area of QCD (both perturbative and soft) are reviewed.
\end{abstract}

Keywords: particle physics, proton proton collisions, LHC, QCD, strong interactions, quarkonium

PACS: $12.38 . t-12.85 . t-13.87 . \mathrm{a}-14.40 . \mathrm{Pq}$

\section{INTRODUCTION}

The most impressive result of the analyses of LHC data collected in the 2011-12 Run 1 is represented by the observation [1] of a scalar particle at a mass of $125 \mathrm{GeV}$ and its unambigous characterization as the messenger of the Brout-Englert-Higgs mechanism. This discovery led to the Nobel prize for Englert and Higgs in 2013.

This was not the only remarkable achievement of Run 1: certainly one can add the observation of the "golden SUSY" channel $B_{s} \rightarrow \mu \mu$ [2] at a rate which is still compatible with the Standard Model (SM) expectation, the evidence of the onset of gluon plasma effects in $\mathrm{Pb}-\mathrm{Pb}$ [3] and, to a lesser extent, also in $\mathrm{p}-\mathrm{Pb}$ collisions. The current datasets from $\mathrm{p}-\mathrm{p}$ collisons, for an integrated luminosity of $\sim 30 \mathrm{fb}^{-1}$ for ATLAS and CMS, $\sim 3 \mathrm{fb}^{-1}$ for LHCb, have allowed stringent tests of SM over a very large number of physics channels. So far, the LHC scientific output represents a triumph of the SM (see Fig. 1, where CMS results are summarized as an example). The theoretical predictions for production crosssections span over more than six orders of magnitude in strength and, within the errors, are in excellent agreement with observations. LHC is just entering an era of precision measurements.

To accomplish this task, it is mandatory to improve our knowledge of QCD behaviour, as the interactions among quarks and gluons permeate the quasi-totality of processes at LHC and therefore practically all measurements done at LHC can be considered as QCD studies. The test of the QCD theory of SM at LHC is performed through the exploration of a new kinematic range never accessed before, where new processes become important (double parton scattering being a striking example among many). Therefore QCD can be probed at many different scales (those of HERA, Tevatron, and LHC), in a variety of initial states (e-p, p-pbar, p-p), and in several domains of $\left(x, Q^{2}\right)$ phase space (see Fig. 2, left).

An increased understanding and modeling of QCD interactions has a direct impact on the potential for precision measurements and discoveries. As an example, the current largest systematics for Higgs cross-section is from $g g$ fusion: $7 \%$ due to QCD scales and $7 \%$ due to the knowledge of parton momentum distribution inside the proton. Moreover, the indetermination on the W/Z+jet event mass shape is often one of the largest uncertainties in backgrounds to t-quark physics, SUSY, Higgs and exotic searches. On the other end, the studies of quarkonium production, spectroscopy of exotic states, and matter at high densities (in $\mathrm{Pb}-\mathrm{Pb}$ and $\mathrm{p}-\mathrm{Pb}$ collisions) can reveal the underlying QCD production mechanisms, and could reserve surprises. Results on spectroscopy and heavy ions are not included in this review.

The talk will briefly discuss the main aspects and phenomenology of QCD physics at LHC, it will report a selected sample of measurements in perturbative QCD, the status of analyses in quarkonium production and, some of the results in the field of soft QCD. The outstanding number of QCD-related measurements forces to choose only a small subset: apologies for any relevant omission. Finally, an overview of the perspectives of LHC and experiment upgrades will also be given.

\footnotetext{
${ }^{1}$ Invited talk at Quark Confinement and Hadron Spectrum XI, September 8-12, 2014, St. Petersburg State University (Russia)
} 


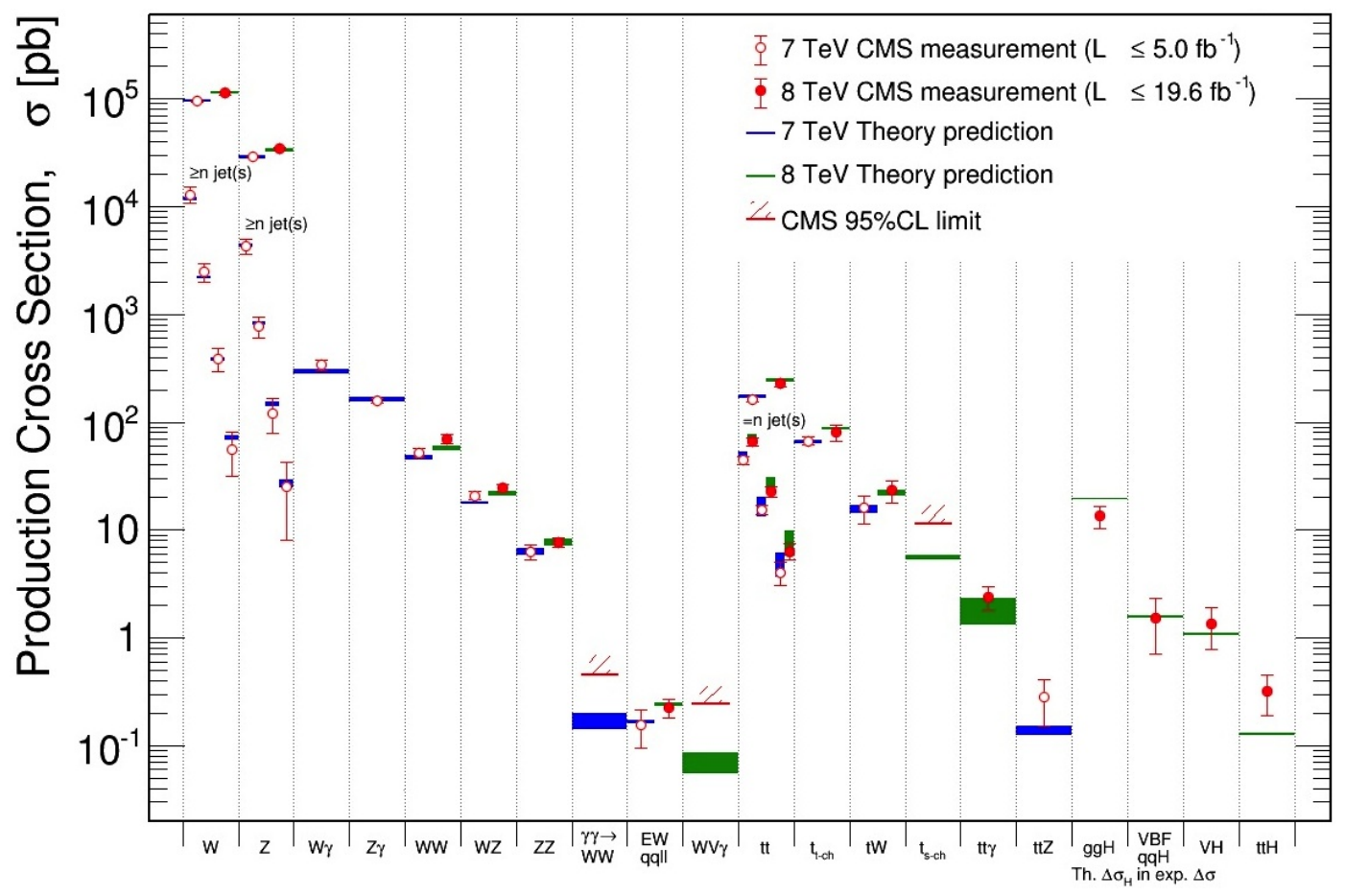

FIGURE 1. Compilation of predicted and measured Standard Model production cross sections for the CMS experiment in electroweak, top and Higgs physics, for Run 1 LHC energies [4].

\section{QCD PHYSICS AT LHC}

QCD can be considered as the main ingredient of any process at LHC. Phenomenology of p-p collisions is determined initially by how valence and sea quarks and gluons interact according to their momentum distribution inside the parton (in QCD terms, the Parton Distribution Functions, PDFs). PDFs are currently evaluated using HERA and fixed target data, but the models that allow extrapolations to the much larger $Q^{2}$ probed at LHC must be tested, bringing hopefully also a reduction of the current systematic uncertainties. When partons and gluons are colliding in the primary interaction, several QCD processes participate to the creation of the final state: the partonic hard crosssections calculated in a pure perturbative regime determine the production rates of specific final states; the quark hadronization and the subsequent parton shower development generate jets and give birth to quark bound states, where a particular interest lies in the study charmonium and bottomonium spectra.

The last two phases are characterized by the strong interplay of perturbative and soft QCD phenomena. Which are the ingredients of QCD predictions? The shapes of PDFs and their evolution in the $\left(x, Q^{2}\right)$ plane, governed by the dynamics of evolution equations, the QCD cross-sections determined by calculations at Leading Order, Nextto-Leading Order and Next-to-Next Leading Order (LO, NLO, NNLO), the MonteCarlo parton shower generators which describe the hadronization and the dynamical models for quarkonium creation. Given the huge amount of data collected at LHC, it is expected that in any of these sectors, the improvement in knowledge will be terrific and the first results are confirming this expectation.

Knowledge of PDFs is one of the fundamental limit for Higgs boson characterization in terms of couplings [5]. In addition to the above mentioned uncertainty on $g g$-fusion process, it is worth to mention the one on the $t \bar{t} H$ associated production (14\%, see Fig. 2, right), and those on various Higgs decay couplings (from $H \rightarrow b \bar{b} \sim 3 \%$ to $H \rightarrow \tau \bar{\tau} \sim$ $6 \%$ ). The same kind of theoretical uncertainties also hold for special Higgs searches with particular cuts (e.g. with jet veto). Moreover, measurements at LHC point to a more complicate proton structure, requesting new corrections to the simple patterns used by theorists in the past.

PDFs are the dominant systematic also for precision measurements like $M_{W}$, that test the internal consistency of SM: half of the current uncertainty on $M_{W}$ is due to the knowledge of PDFs. They are also essential to evaluate the sensitivity in the searches for new particles: as examples, SUSY particle production cross-sections depend strongly on 

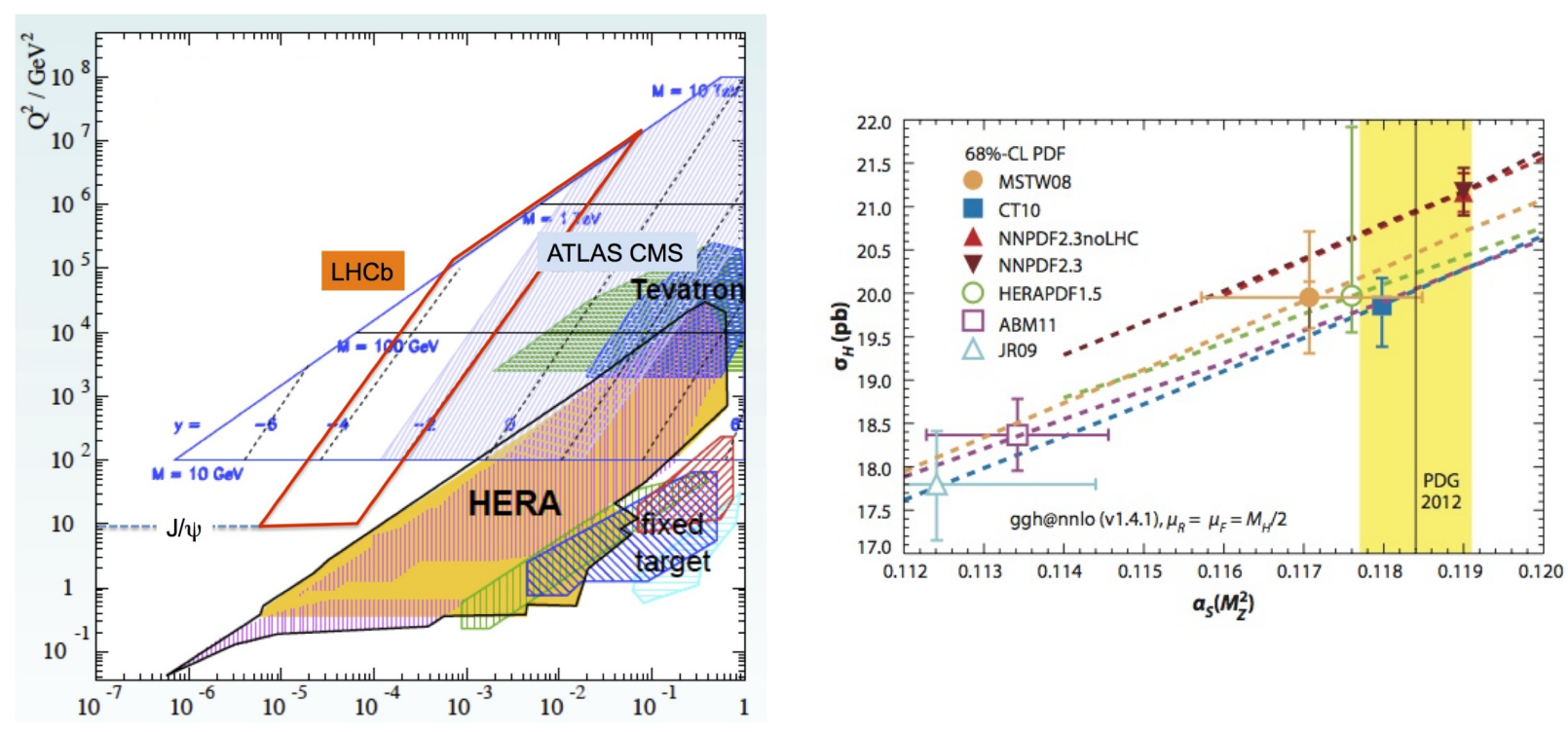

FIGURE 2. (left) Regions in $\left(x, Q^{2}\right)$ variables accessible by the different experimental facilities. (right) Production cross section of $g g \rightarrow H$ at LHC, as a function of the value of the $\alpha_{S}\left(M_{Z}\right)$ coupling constant, and for various parametrizations of PDFs [6].

these functions as well as the evaluation of shapes of invariant di-lepton or di-jet mass spectra. PDFs are also a critical ingredient for evaluating backgrounds in searches for new physics (e.g. $t \bar{t}$ production cross section determination).

\section{PERTURBATIVE QCD MEASUREMENTS AT LHC}

\section{Jet and photon production}

Proton-proton collisions leading to events with high transverse momentum jets are described by perturbative QCD (pQCD) through elementary parton-parton scattering. A fundamental quantity that can be measured and predicted within $\mathrm{pQCD}$ is the double-differential inclusive jet cross section as a function of the jet transverse momentum $p_{T}$ and the absolute jet pseudorapidity $|\eta|$. ATLAS and CMS Collaborations have carried out these measurements at 7 and $8 \mathrm{TeV}$ [7]. LHC data span 16 orders of magnitude in cross-section intensity and up to a $p_{T}$ value of $\sim 2 \mathrm{TeV}$ (see Fig. 3, left). Perturbative QCD is able to describe correctly the data over a wide range of jet transverse momentum and rapidity and over many orders of magnitude in cross section. Some differences between the predictions employing various PDF sets and the data are observed, all of which are qualitatively explained by uncertainties in the low $p_{T}$ region.

The determination of the cross-section for multi-jet production is another important test of pQCD. This measurement is also sensitive to resonances and new interactions, and can be used in searches for physics beyond the SM. The results can be also exploited to study the partonic structure of the proton. In particular, the high dijet-mass region can be used to constrain the PDF of the gluon in the proton at high momentum fraction (Bjorken variable $x$ ). Data are compared to many different PDFs and with different jet sizes. Ratios of cross-sections are also very useful to disentangle several systematic uncertainties. Moreover, ratio of the observed number of 3-jets to 2-jets (a classical way to determine the strong coupling costant $\alpha_{s}$ since PEP/PETRA/LEP times) and the 3-jet invariant mass spectrum measurements determine $\alpha_{s}$. The most recent CMS result [9] $\alpha_{S}\left(M_{Z}\right)=0.1185 \pm 0.0019(\exp ) \pm 0.0028(P D F) \pm 0.0004(N P) \pm_{0.0022}^{0.0055}($ scale $)$ represents an impressive test of pQCD up to the TeV scale (see Fig. 3, right).

The quality of LHC data is such to enter already in the combinations for PDFs determination and this is relevant for consistency checks and for the evaluation of systematics. Fits performed including LHC data prefer a harder spectrum for gluons with respect to the one obtained with HERA-only measurements (see Fig. 4). 
Photons represent a clean and uncolored probe to study the underlying parton-parton interactions. At LHC energies, direct Compton production $(q g \rightarrow q \gamma)$ is dominant $(\sim 70 \%)$ with respect to annihilation $(q \bar{q} \rightarrow g \gamma)$ or fragmentation channels. The differential cross-section as a function of $E_{\gamma}$ energy is used to test pQCD predictions, providing useful information on gluon PDFs. In the domain of events with photons in the final state, one has to mention the importance of $\gamma+$ jet studies, a test of color dynamics, and the isolated $\gamma \gamma$ pair production, which represents a relevant background for $H \rightarrow \gamma \gamma$ analyses.

\section{Vector Boson production associated to jets or heavy quarks}

With the large data sample accumulated at the LHC, detailed investigations of pQCD and electroweak (EWK) effects are now possible over five orders of magnitude in the cross section as a function of jet multiplicity and six orders of magnitude as a function of the jet transverse momenta. During the last few years, advances in the theoretical frameworks for the calculation of final states containing a vector boson and jets allow cross sections to be determined at next-to-leading order (NLO) in pQCD for vector bosons with up to five jets in the final state. Processes with W/Z+jets are also the main background to the production of other, much more rare, SM processes, such as $t \bar{t}$ and single top production. They are also major backgrounds to searches for the Higgs boson and a variety of physics processes beyond the SM. For the production of a massive gauge boson accompanied by jets, jet transverse momenta up to 1 $\mathrm{TeV}$ are now, for the first time, accessible and allow the study of a kinematic region where higher order EWK effects can become as important as those from higher-order pQCD corrections. In this field, ATLAS and CMS are the main players, but LHCb also contributes: Z+D mesons, Z+jets, and in complementary acceptance regions. Therefore only in the combination of data the full LHC potential can be explored. Searches for phenomena beyond the SM are often limited by the uncertainty on the theoretical cross-section for $\mathrm{W}$ (and Z) + jets processes at high momentum scales and large jet multiplicities and it is therefore crucial to perform precision measurements of W+jets production at the LHC.

The data [11] are compared to a variety of theoretical predictions, at both leading order and next-to-leading order, including the use of MC generators and show quite good agreement. However there is currently no theoretical prediction that is able to provide an accurate description of the data for all measured distributions. The best agreement is obtained using generators based on NLO+parton shower.

The study of associated production of a $\mathrm{W}$ boson and a c-quark at LHC provides direct access to the s-quark content of the proton at an energy scale of the order of the W-boson mass $Q^{2} \sim(100 \mathrm{GeV})^{2}$. This sensitivity is due to the dominance of $s g \rightarrow c W^{-}$diagrams at the hard-scattering level, while the Cabibbo suppressed reaction initiated by a d-quark contributes about $\sim 10 \%$. The large production rates available at the LHC provide the first opportunity for a measurement with sufficient precision to constrain the s-quark PDF at $x \sim 0.01$. First data from ATLAS and CMS, included in the determination of s-quark PDFs, show (see Fig 5) different levels of suppression of this quark in
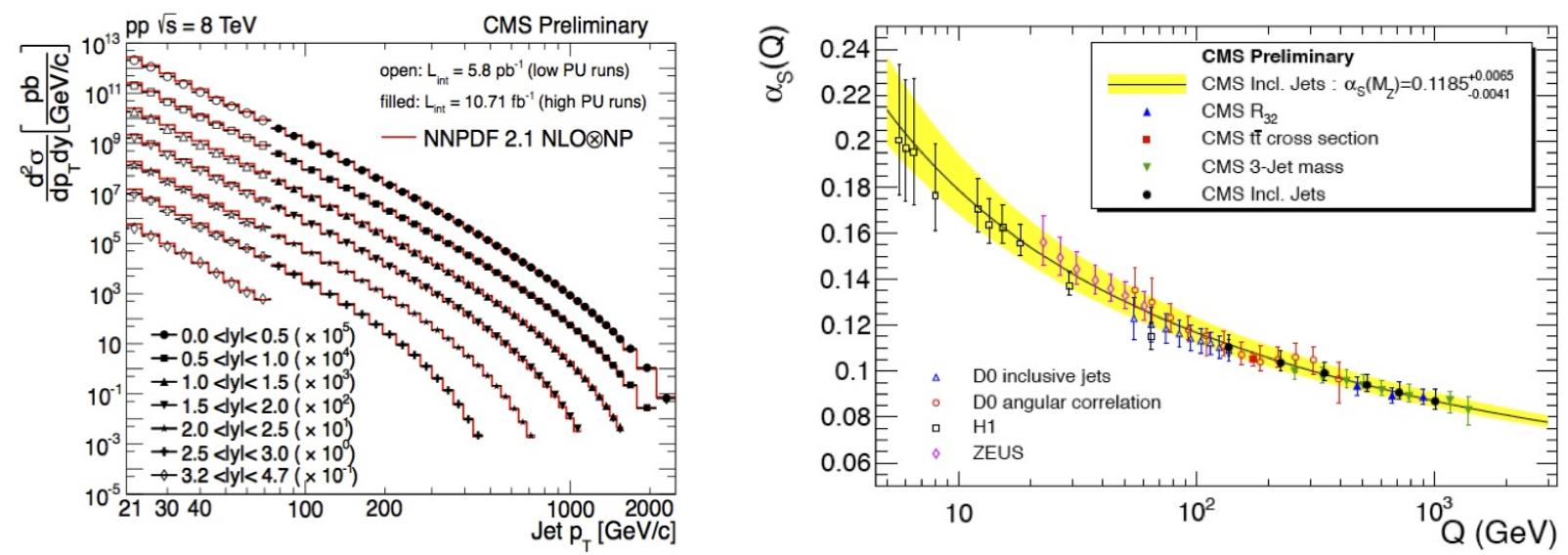

FIGURE 3. (left) Double differential cross-section for inclusive production of single jet measured in CMS. (right) Measurement of $\alpha_{s}$ strong coupling constant in CMS. 

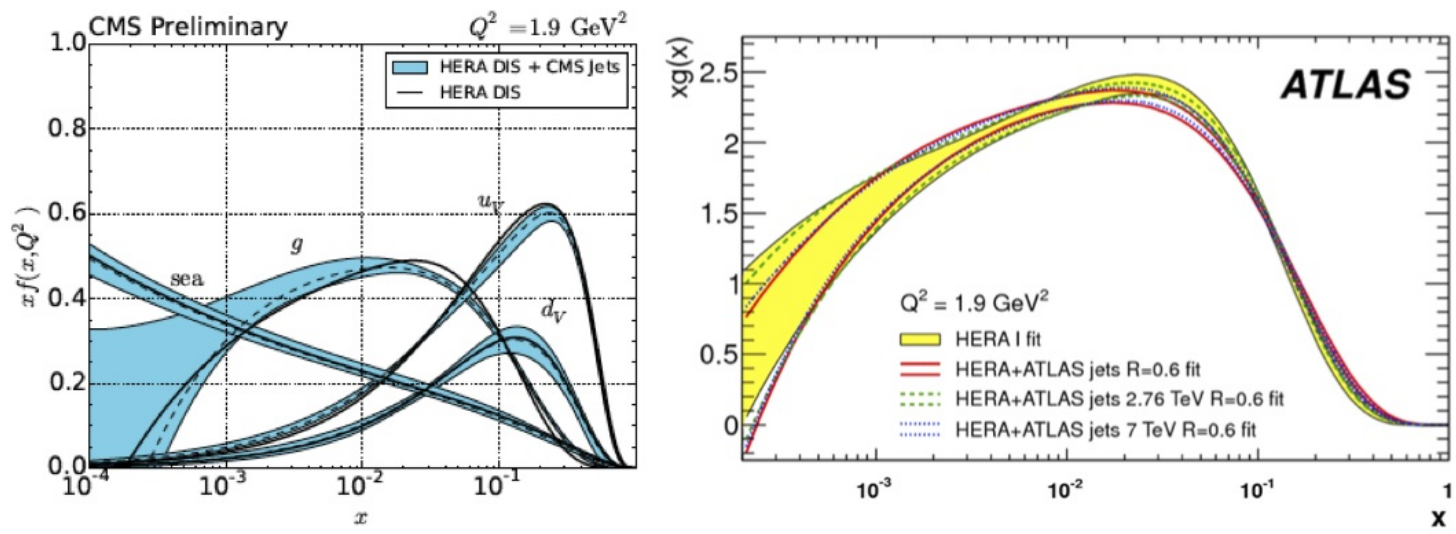

FIGURE 4. Parton and gluon distribution functions obtained from fit including ATLAS [8] and CMS [9] inclusive jet crosssection distributions.

comparison to the light quark sea: ATLAS data do not support this suppression, while CMS one are still compatible with a reduction of the s-quark content, as observed in fits from HERA and deep inelastic scattering experiments. Future data with higher statistics will hopefully solve this apparent discrepancy.

\section{W lepton charge asymmetry - Drell Yan production}

Measurements of the production cross-section of electroweak bosons constitute an important test of the Standard Model at LHC energies. Theoretical predictions, which are available at NNLO order in pQCD rely on the parametrisations of the partons inside the proton. At LHC the study of $W \rightarrow$ lepton charge asymmetry allow a precise determination of the up/down valence quark ratio and sea $\bar{q}$ densities in the proton, as experimental and theoretical uncertainties partially cancel. The different acceptances in ATLAS/CMS and LHCb determine different probed regions of the momentum fraction: central $\left(10^{-3}<x<10^{-1}\right)$, and forward $\left(10^{-4}<x<10^{-3}\right)$. The uncertainties on the proton PDFs are larger at smaller values of $x$ variable. It is worth to notice that asymmetry changes sign in the LHCb acceptance. Results [14] are compared to Standard Model predictions calculated at NNLO in perturbative QCD, which agree well with the data. LHC data provide constraints on the determinations of PDFs, with a total uncertainty that is comparable with, or in some cases better, than the uncertainty on the theory calculations (see Fig 6, left).
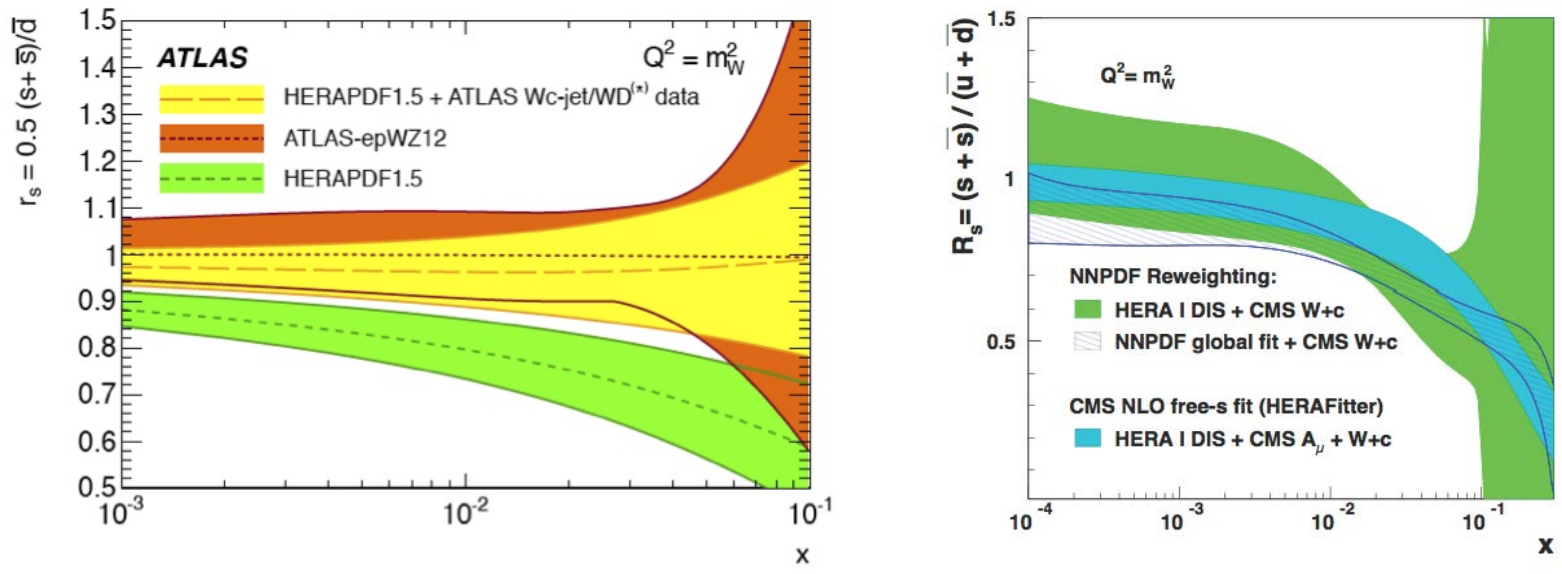

FIGURE 5. Measurements of the ratio of strange to light quarks, as a function of $x$ variable in ATLAS [12], and CMS [13]. 

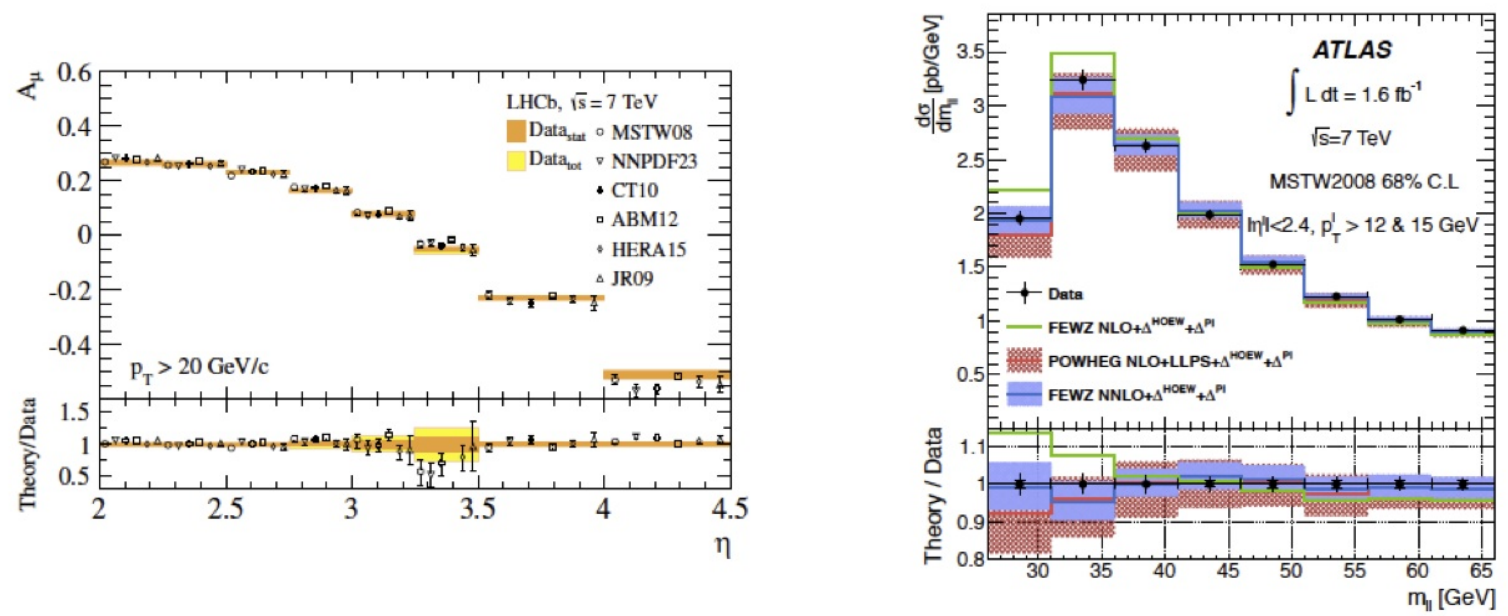

FIGURE 6. (left) $W \rightarrow$ lepton charge asymmetry in bins of muon pseudorapidity with LHCb data. (right) Drell-Yan dilepton distribution at low masses in ATLAS.

The Drell-Yan process of dilepton production in hadronic interactions provides important information on the partonic structure of hadrons. Measurements in the region of low [15] and high [16] dilepton invariant mass, provide complementary constraints on up/down and anti-quarks. Additionally, the production of DY lepton pairs is a source of background for other SM measurements, considering that the mass spectrum may be modified by new physics phenomena. The results are supported by a QCD analysis of the measurements performed at NLO and NNLO. The PDFs are fitted to the new measurements together with inclusive e-p measurements from HERA. The NNLO fit performs significantly better than the NLO one in describing the low mass data (see Fig. 6, right)

\section{Double parton scattering}

The model in which a parton of one nucleon interacts only with a single parton of the other nucleon is only an idelization of p-p collisions. In reality, the interaction is much more complex and can involve many partons (MPI, Multiple Particle Interaction). An extremely important aspect of MPIs is represented by the double parton scattering (DPS), where two simultaneous hard interactions happen, which can be considered as the "hard component" of a general softer behaviour comprising MPIs. DPS has been observed with extremely low statistics at ISR, SPS, Tevatron, but at LHC it is expected to be non negligible $(\sim 10 \%$ at low $x<0.2)$ and even larger in Run 2 due to higher energy. The evaluation of this kind of process is relevant in searches for new physics.

The variety of measurements at LHC that bring information on DPS is very large: $\mathrm{J} / \psi+$ charm and double charm production [17]; double $\mathrm{J} / \psi$ production [18]; double $\mathrm{J} / \psi$ production in CEP events [19]; Z+charm production [20], $\mathrm{W}+\mathrm{J} / \psi$ production [21], and $\mathrm{W}+2 \mathrm{j}$ production [22] and from fits to inclusive $D^{0}$ production [23].

\section{LHC and the PDFs: a recap}

The recent results from LHC data are now entering into PDF fits, not only in areas which are traditional for hadron colliders, but also, due the increased statistics available, in channels so far not used due to rarity of phenomena. The current status of LHC data entering in PDFs [24] can be summarized, first recalling the "historical" measurements entering PDF fits: inclusive jet production, W lepton asymmetry and low/high mass Drell-Yan spectra.

In addition to these channels, some very interesting "new entries" (some of them discussed in the paragraphs above), are appearing, thanks to LHC energy and statistics:

- measurement of t-quark pair production cross-section, constraining gluon PDF at large $x$;

- study of W+charm events, sensitive to s-quark PDF; 

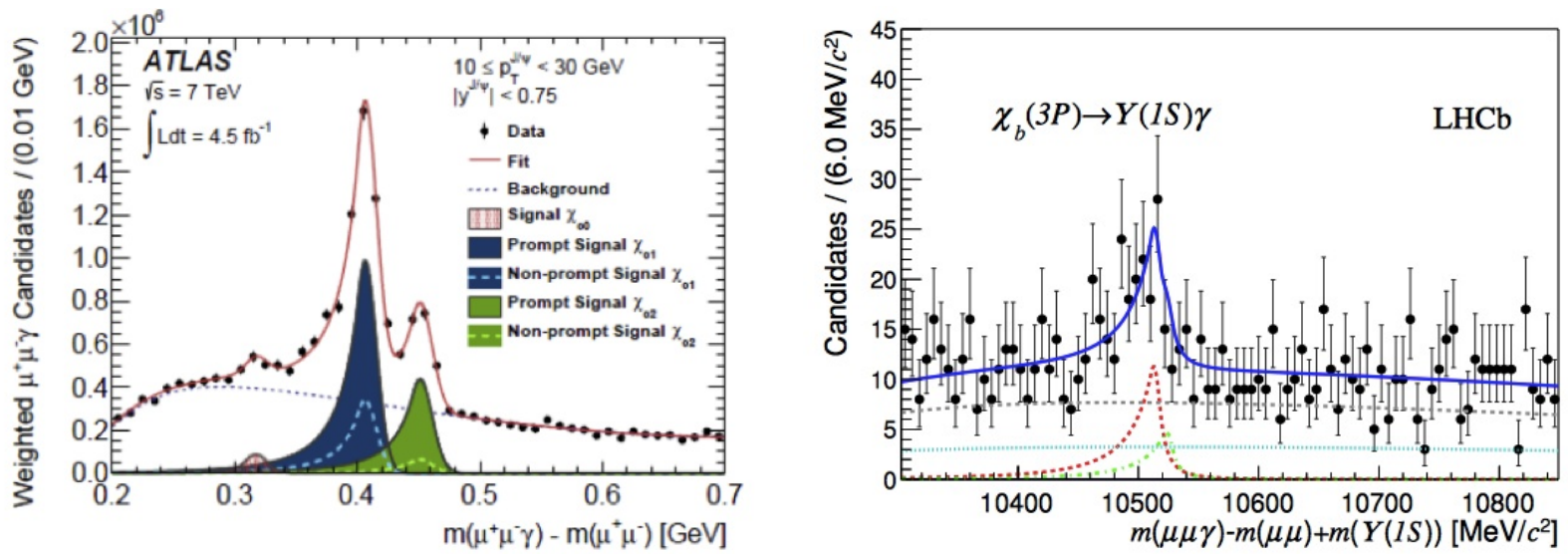

FIGURE 7. (left) Invariant mass distribution of the $\chi_{c 1, c 2} \rightarrow \mathrm{J} / \psi \gamma$ decays in ATLAS [26]. (right) Invariant mass distribution of the $\chi_{b 1}(3 P) \rightarrow \Upsilon(1 S) \gamma$ decays in LHCb [27].

- observation of high $p_{T} \mathrm{~W}$ and $\mathrm{Z}$ production, sensitive to gluon and $d / u$ ratio in PDF;

- identification of final states with isolated $\gamma$, probe for gluon PDF.

It is evident that in the near future, other interesting observables from LHC will enter the PDFs fits: data from vector bosons + jets, Z+charm and Z+beauty for gluon, c-quark and b-quark PDFs and single top production for gluon, c-quark and b-quark PDFs.

\section{QUARKONIUM PRODUCTION AT LHC}

LHC is a heavy quarkonium factory, useful to study how QCD binds colorful quarks in hadrons, a mixture of pQCD and soft QCD effects well separated in time. Historically, quarkonium production has been described using a Color Singlet Model (CSM) which is fully predictive, but was found to be unable to describe Tevatron data on $\mathrm{J} / \psi$ and $\psi(2 S)$ production cross sections. Therefore a non-relativistic QCD effective theory which foresees a superposition of color octets states (NRQCD "Color Octet Model", tuned with Tevatron collider data) has replaced the naive CSM. In this theoretical framework, the huge amount of LHC data covers precise measurements in

$\cdot c \bar{c}, b \bar{b}$ states production cross sections of prompt $\mathrm{J} / \psi, \psi(2 S), \chi_{c n}, \Upsilon(n S)$, and $\chi_{b n}$ mesons;

- momentum and transverse momentum spectra of the above states;

- evaluation of contributions from feed-down effects (i.e. those particles not produced directly, but obtained from cascade decays of other prompt states);

- study of polarization effects.

LHC experiments [25] have shown excellent capabilities in identifying quarkonium in p-p collisions, thanks to the performance of vertex, tracking and electromagnetic calorimeter systems: prompt $\mathrm{J} / \psi$ decaying in di-muons are well separated from those originating from B decays, the $\Upsilon(n S)$ family elements are resolved and decays with photons in final state are detected, with a particular challenge due to the very near resonances, as $\chi_{c 1, c 2}(1 P) \rightarrow \psi(n S) \gamma$, and $\chi_{b 1, b 2}(1 P) \rightarrow \Upsilon(n S) \gamma($ see Fig. 7).

High statistics $p, p_{T}$ spectra both at small/high momentum are now available for a variety of quarkonium states: ATLAS and CMS in the high $p_{T}$ region, LHCb and ALICE in forward direction, at low $p_{T}$. For comparison with theory, it is particularly important to access large $p_{T}$, being low $p_{T}$ region affected by non perturbative effects. The large amount of data on quarkonium spectra shows an excellent agreement with NRQCD Tevatron tuned predictions across a wide range of species and rapidities. CSM predictions even corrected to higher orders, still partially agrees with data (see Fig 8, left). Moreover, to obtain a full view of quarkonium production mechanism, it is crucial to understand the amount of feed-down contributions and the relative rates of production of higher order excited states. As an example, feed-down contribution to $\mathrm{J} / \psi$ production from $\psi(2 S)$ is measured to be $\sim 8 \%$ and from $\chi_{c} \sim 20 \%$. The experimental observation made at LHC that S-wave and P-wave quarkonia resonances have rather similar spectra 

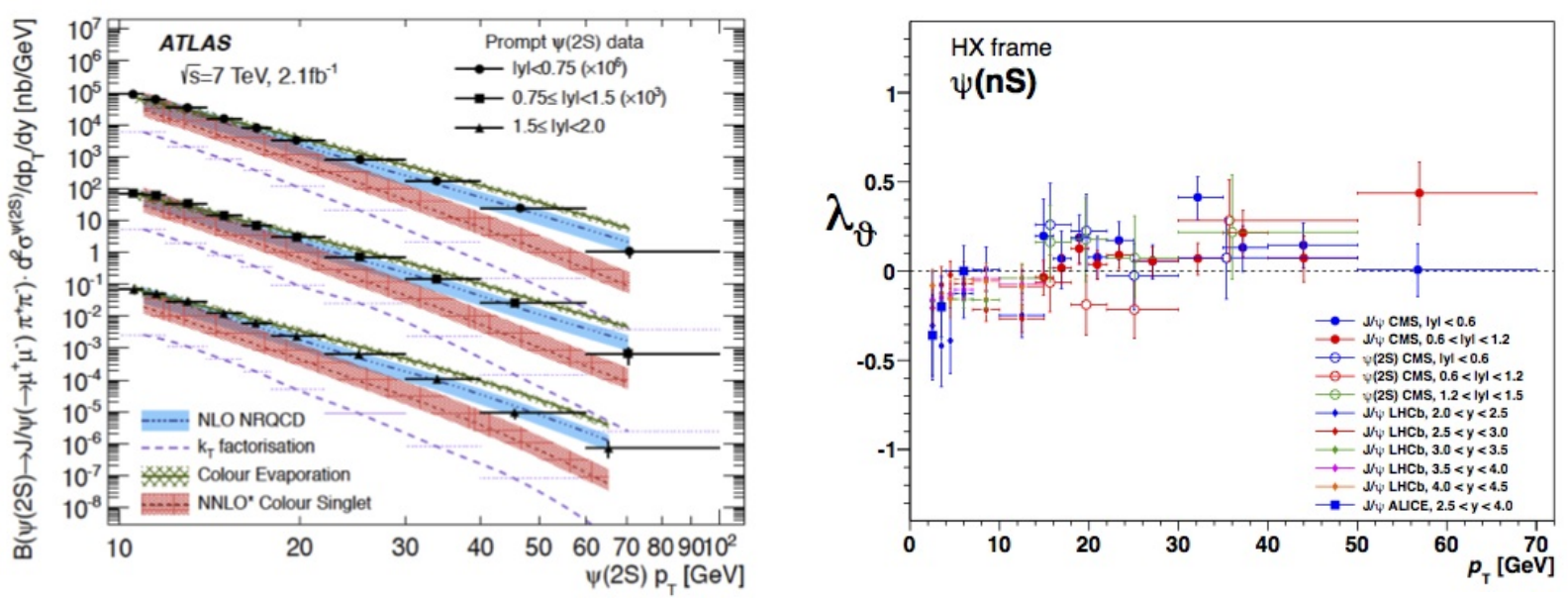

FIGURE 8. (left) Transverse momentum spectrum of prompt $\psi(2 S)$ in ATLAS [28]. (right) Compilation of charmonium states polarization values as measured in LHC experiments [29].

(at large $p_{T}$ ), points to a similarity of their production mechanism, excluding some of the model proposed (e.g. $k_{T}$-factorization approach).

A stringent test of any model for quarkonium production is represented by the measurement of polarization. NRQCD effective theory predicts non-zero polarization values for most of quarkonium states. From the experimental point of view, measuring polarization is quite challenging, as it is mandatory to determine precisely the absolute efficiencies and acceptances of the detector and the measurements are affected by the systematics due to unknown polarization and feed-down effects. So far, no sign of polarization has been observed (see Fig 8, right).

This result has suggested [29] that the un-polarized octet element $1 S_{0}^{[8]}$ could have a dominant contribution in the final quarkonium states, while tiny terms could be given by the polarized elements. It has also been also proposed [29] that the current procedure of tuning of NRQCD using $p, p_{T}$ data and then predicting polarization values, can be changed in a more global fit approach where spectra and polarization value are fitted all together to get the parameters of the theory. The future increase in statistics of LHC data will help certainly to move in this direction.

\section{SOFT QCD STUDIES}

As already mentioned, non perturbative QCD represents a very large part of our understanding of strong interactions and of collision process at LHC. The goal of soft QCD studies is to improve our (imperfect) modeling of strong interactions. These include the modes of transition of hard/soft regimes, the mechanisms of diffractive processes, the hadronization and the confinement, the search for new QCD dynamics, the tuning of MC generators, the interplay between collider and the cosmic ray physics.

Several different tools are available for studying soft QCD effects at LHC: the analysis of charged particle multiplicities and energy flow, the study of diffractive scattering and Central Exclusive Production, the observation of multi-parton interactions (MPI) and underlying event (UE), the measurement of total p-p cross-section, and many others. Due to lack of time only few examples will be briefly discussed.

\section{Diffractive and elastic events - Central exclusive production}

Perturbative parton-parton hard collisions account for $\sim 60 \%$ of total activity at LHC, the rest being diffractive and elastic collisions, mainly dominated by non-perturbative QCD (see Fig 9, left). Among those events, an interesting class is represented by the central exclusive production (CEP) one, elastic or single inelastic, defined as processes of the type $p p \rightarrow p X+R$, where $\mathrm{X}$ is a fully measured on-shell state such as quarkonium, jet-jet system, gg, $\mathrm{H}$, etc ... and " $R$ " represents a large rapidity-gap in the detector. In fact the signature of these events is the production of very few 

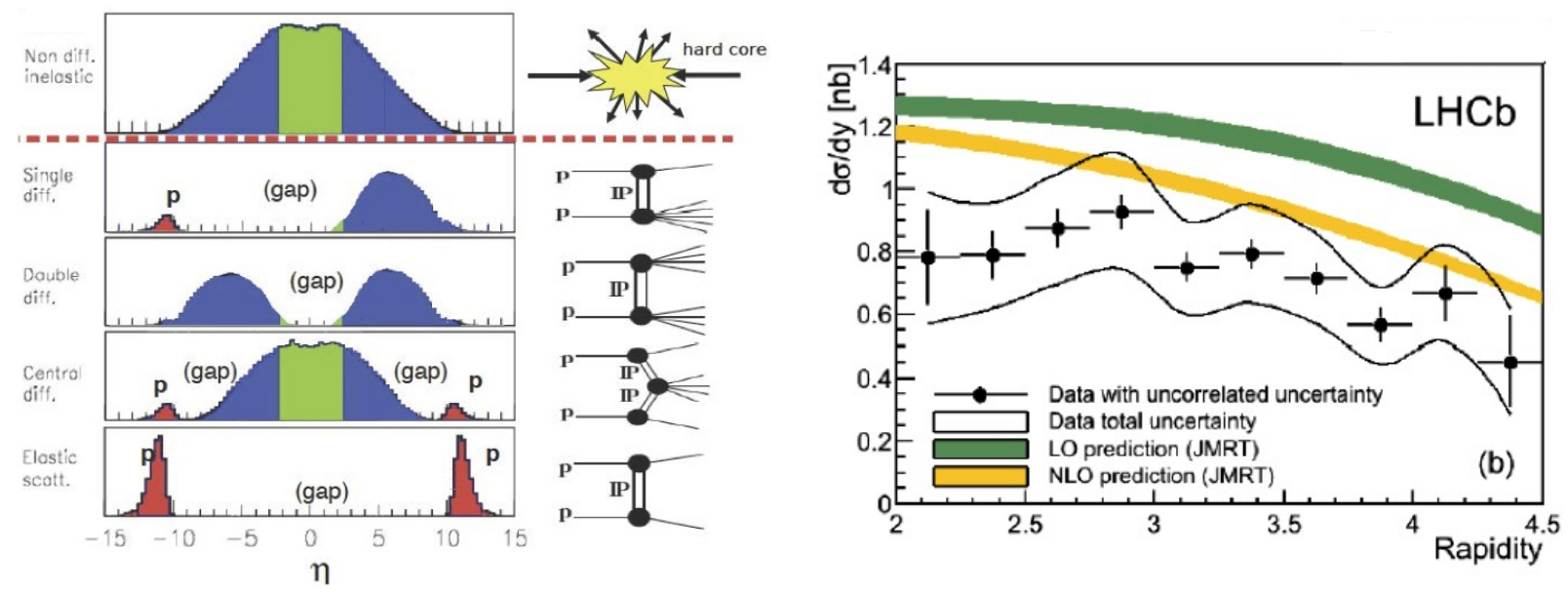

FIGURE 9. (left) A schematic representation of activity in LHC detectors as a function of pseudorapidity in inelastic events (top strip) and in various topologies of diffractive events (strips below). Drawing adapted from [30]. (right) Cross-section for $\psi(2 S)$ central exclusive production as a function of rapidity in $\mathrm{LHCb}$.

particles in the detector and the presence of large empty regions in the acceptance. As an example, central exclusive Higgs production has attracted significant experimental and theoretical interest for various important reasons, as to a very good approximation, in CEP the primary active di-gluon system obeys a $J_{z}=0$, C-even, P-even, selection rule (where $J_{z}$ is the projection of the total angular momentum along the proton beam axis). Therefore this class of phenomena offers interesting opportunities for SM tests or for the exclusive production of specific states in particular and experimental favorable conditions.

Two are the examples that can be picked-up on recent analyses on CEP events at LHC: the exclusive WW production in CMS [31], which allows the determination of anomalous quartic gauge boson couplings in $\gamma \gamma \rightarrow W W$ events, and the exclusive production in $\mathrm{LHCb}$ [19] of single and double $\mathrm{J} / \psi, \psi(2 S)$ (Fig 9, right), with di-muons in the final state. The exchange of colorless objects $(\gamma$, pomeron) in single elastic diffractive process (when a proton disappears in the beam pipe), probe $x$ variable up to $510^{-6}$.

$\mathrm{LHCb}$ is particularly suited to study CEP events: excellent tracking and particle identification (PID) capabilities up to high $\eta(\sim 4.5)$, low pileup conditions (this aspect is critical and cannot be easily met in ATLAS and CMS without a strong reduction of available statistics), the implementation of a special trigger with high efficiency for low multiplicity events with low $p_{T}$ tracks. Searches for exclusive production of X(3872), open charm, and di-mesons, using forward PID capabilities to detect hadrons in the final states, are also ongoing. Moreover, LHCb has excellent perspectives in this sector for Run 2 due to the implementation of a new detection sub-system (HERSCHEL) capable of vetoing protons with activity within $5<|\eta|<8$.

\section{Inelastic, elastic and total proton-proton cross-sections}

The interaction cross-sections of p-p collisions are fundamental quantities not computable in pQCD but bound from basic principles of Quantum Mechanics: the Froissart limit, the optical theorem, the dispersion relationships. Any deviation from the expected behaviour would appear as a sign of emerging new physics.

Inelastic, elastic and total p-p cross-sections have been measured by several experiments at LHC: ALICE, ATLAS, CMS and TOTEM. The most recent and precise measurement result has been obtained by ATLAS [34] $\sigma_{t o t}=95.4 \pm 1.8 \mathrm{mb}$, in agreement with expected fits from lower energy data (see Fig. 10 ).

LHC analyses show an interesting complementarity with cosmic ray data obtained by the largest Extended Air Shower arrays (EAS, such as Auger) which can detect cosmic primary energies well above LHC. However, the energy determination of these ultra high energy showers can be obtained only via the comparison with hadronic shower Monte Carlo: in this respect, LHC can help in tuning the simulation. LHC experiments contribute to this effort with several measurements, in particular with forward energy flow studies. It must be noticed the remarkable agreement of data at high $\eta$ [35] with the simulation obtained with EAS Monte Carlo (Epos-Sybill) which are not tuned to LHC data. 


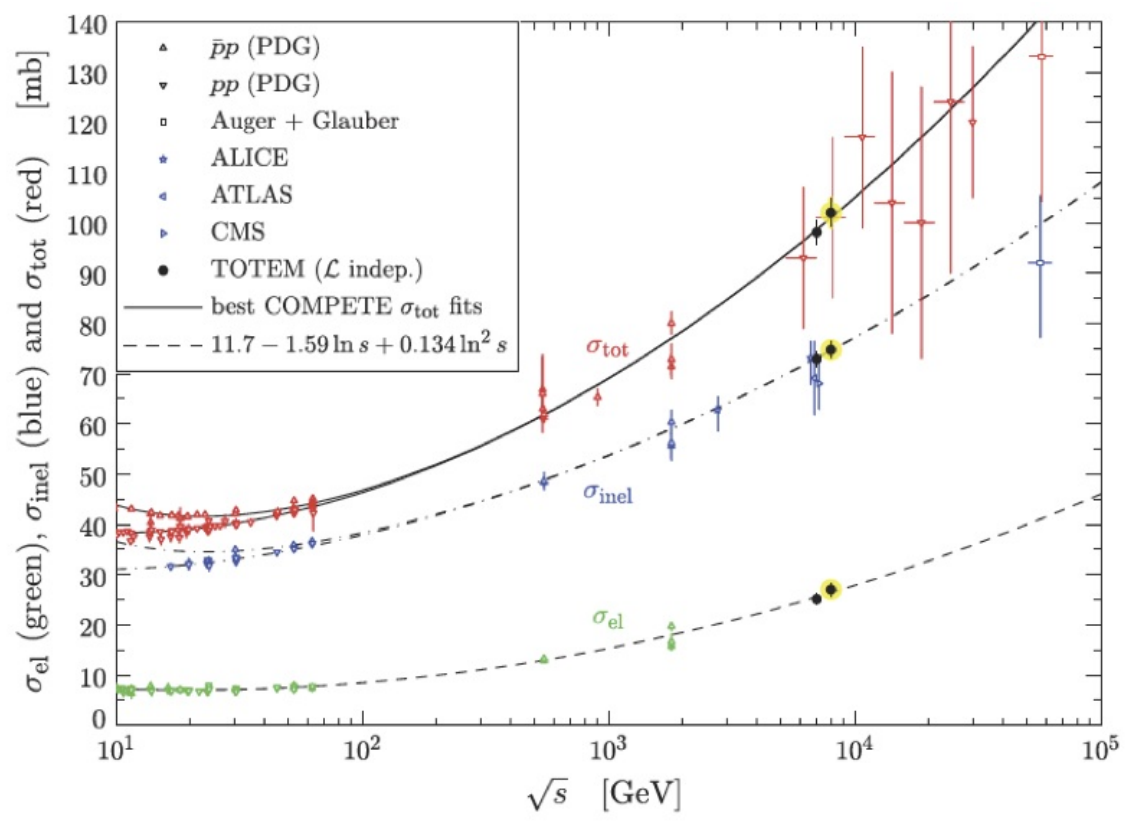

FIGURE 10. Compilation of elastic, inelastic and total p-p cross-sections as a function of centre-of-mass energy [33].

\section{FUTURE PERSPECTIVES AT LHC RUN 2 AND BEYOND}

LHC experiments are now completing the refurbishment of detectors, that started at the beginning of the current long shutdown no.1 (LS1). They are now ready to take data again in Run 2 (2015-mid 2018). LHC operation conditions will have, in addition to a centre-of-mass energy of $13 \mathrm{TeV}$, a $25 \mathrm{~ns}$ beam structure and a planned luminosity (for p-p collisions) of $1.510^{34} \mathrm{~cm}^{-2} \mathrm{~s}^{-1}$ for ATLAS and CMS, to collect and integrated luminosity of $\sim 100 \mathrm{fb}^{-1}$, and $\geq 410^{32} \mathrm{~cm}^{-2} \mathrm{~s}^{-1}$ in LHCb, for a total of $7 \mathrm{fb}^{-1}$ expected. The upgrades of ALICE and LHCb and those of ATLAS and CMS, relative to Phase-1, will be installed during long shutdown no.2 (LS2, mid 2018 - end 2019).

In Run 3 (2020-2022) the running conditions will foresee the increase of LHC peak luminosities up to $2.210^{34} \mathrm{~cm}^{-2} \mathrm{~s}^{-1}$ in ATLAS and CMS, to collect an integrated luminosity of $\sim 500 \mathrm{fb}^{-1}$. Thanks to the installed upgrades, LHCb will operate at higher luminosity $\left(210^{33} \mathrm{~cm}^{-2} \mathrm{~s}^{-1}\right)$ and ALICE will handle a substantially higher trigger rate. During long shutdown no.3 (LS3, 2023 - 2025) it is planned to install the phase-2 upgrades of ATLAS and CMS and the necessary elements for LHC to operate at High Luminosity (HL-LHC). New tracking detectors will be capable of dealing with high radiation doses and high pile-up (average $\sim 140$ ). Extended forward tracking devices are foreseen, while new track and calorimetry real-time triggers and largely upgraded DAQ systems will be installed.

In Run 4 (2026-2028), and in Run 5 (2030-2032), LHC should be able to deliver a peak luminosity of $510^{34} \mathrm{~cm}^{-2} \mathrm{~s}^{-1}$ with an "ultimate luminosity performance" set at $710^{34} \mathrm{~cm}^{-2} \mathrm{~s}^{-1}$, to permit the detectors to integrate $3000 \mathrm{fb}^{-1}$, or more, up to year $\sim 2035$.

\section{CONCLUSIONS}

Soft and perturbative QCD studies represent a substantial part of LHC activities: a better understanding of QCD is a priority for any test of SM (in particular for Higgs precision physics) or for new physics searches. LHC experiments offer an unprecedented opportunity in terms of phase space accessible in the $\left(x, Q^{2}\right)$ variables, of statistics, of data quality. Analyses are quite complex, therefore the full data set of Run 1 has not been fully exploited yet. Run 1 represents only a small "appetizer" of the many potentialities available: LHC data start to be precise enough to constrain PDFs, and a wide range of new processes that provide info on PDFs are now available at LHC. Run 2 will increase by more than a factor four, not considering the effect of energy, the statistics available, which is relevant 
for the rare processes. Detectors have been upgraded and refurbished during LS1 and are ready for collisions at 13 $\mathrm{TeV}$. Even more brilliant prospects are expected for Run 3 and beyond, when the machine (HL-LHC) and the detectors will be substantially upgraded.

\section{ACKNOWLEDGMENTS}

I thank the organizers of this very interesting Conference for the kind invitation. I acknowledge the help and the many suggestions of G. Manca, V. Belyaev, A. Tricoli. I thank in particular V. Belyaev for his careful reading and the useful comments on this manuscript.

\section{REFERENCES}

1. $\quad$ ATLAS Collaboration, Phys. Lett. B716 (2012) 1; CMS Collaboration, Phys. Lett. B716 (2012) 30.

2. $\quad$ CMS Collaboration, Phys. Rev. Lett. 111 (2013) 101804; LHCb Collaboration, Phys. Rev. Lett. 111 (2013) 101805

3. ALICE Collaboration, Phys. Rev. Lett. 109 (2012) 072301

4. A. David, invited talk at ICHEP 2014

5. J. Campbell, invited talk at ICHEP 2012

6. S. Forte, G. Graeme, Annu. Rev. Nucl. Part. Sci. 63 (2013) 291

7. ATLAS Collaboration, Phys. Rev. D86 (2012) 014022, CMS Collaboration, CMS-PAS-SMP-2012-012 and CMS-PAS-FSQ2012-031

8. $\quad$ ATLAS Collaboration, Eur. Phys. J. C73 (2013) 2509

9. CMS Collaboration, $C M S-P A S-S M P-2012-028$

10. ATLAS Collaboration, JHEP 05 (2014) 059

11. ATLAS Collaboration, ATLAS-CONF-2014-035; CMS Collaboration, CMS-PAS-SMP-2012-023

12. ATLAS Collaboration, JHEP 05 (2014) 068

13. CMS Collaboration, JHEP 02 (2014) 013

14. ATLAS Collaboration, Phys. Rev. D85 (2012) 072004; CMS Collaboration, Phys. Rev. D90 (2014) 032004; LHCb Collaboration, arXiv: 1408.4354

15. ATLAS Collaboration, JHEP 06 (2014) 112; CMS Collaboration, JHEP 10 (2011) 132; LHCb Collaboration, JHEP 06 (2012) 058 and JHEP 02 (2013) 106

16. ATLAS Collaboration, Phys. Lett. B725 (2013) 223; CMS Collaboration, JHEP 12 (2013) 030

17. LHCb Collaboration, JHEP 06 (2012) 141 and JHEP 03 (2014) 108

18. LHCb Collaboration, Phys. Lett. B707 (2012) 52; CMS Collaboration, CMS-PAS-BPH-2011-021

19. LHCb Collaboration, J. Phys. G41 (2014) 115002

20. LHCb Collaboration, JHEP 04 (2014) 91

21. ATLAS Collaboration, JHEP 04 (2014) 172

22. ATLAS Collaboration, New J. Phys. 15 (2013) 033038; CMS Collaboration, JHEP 03 (2014) 32

23. ALICE Collaboration, JHEP 01 (2012) 128); R. Maciula and A. Szczurek, Phys. Rev. D87 (2013) 074039

24. J. Rojo, invited talk at SM@LHC2014; M. Ubiali invited talk at LHCP 2014

25. H. Woehri, invited talk at ICHEP 2014, for a recent review of quarkonium physics and references on measurements at LHC

26. ATLAS Collaboration, JHEP 07 (2014) 154

27. LHCb Collaboration, JHEP 10 (2014) 88

28. ATLAS Collaboration, JHEP 09 (2014) 79

29. P. Faccioli et al., arXiv:1403.3970

30. D. d'Enterria, arXiv:0806.0883

31. CMS Collaboration, JHEP 07 (2013) 116

32. ATLAS Collaboration, JHEP 04 (2014) 172

33. TOTEM Collaboration, Phys. Rev. Lett. 111 (2013) 012001

34. ATLAS Collaboration, Nucl. Phys B889 (2014) 486

35. ATLAS Collaboration, JHEP 11 (2011) 033; CMS Collaboration, JHEP 11 (2011) 148; LHCb Collaboration, Eur. Phys. J. C73 (2013) 2421 